\title{
ON DIVERGENT LACUNARY TRIGONOMETRIC SERIES
}

\author{
L. THOMAS RAMSEY
}

ABSTRACT. Let $S=\left\{\lambda_{n}\right\}_{n=1}^{\infty}$ be a sequence of positive real numbers such that $\lambda_{n+1} / \lambda_{n} \geqslant q>1$ for all $n$. If $q>8$ then any divergent series with frequencies in $S$ has its real part diverging (uniformly) to $+\infty$ on a set of positive logarithmic capacity. It is necessary that $q>2$. A new sufficient condition for the generalized capacity of a set to be positive is developed and then applied in the proof.

In [3] Csordas, Lohwater and Ramsey prove the following theorem. We use $\exp (t)$ to denote $e^{i t}$.

THEOREM 1. Let $S=\left\{\lambda_{n}\right\}_{n=1}^{\infty}$ be a set of positive real numbers such that $\lambda_{n+1} / \lambda_{n} \geqslant$ $q>1$ for all $n$. Let $\sum_{n=1}^{\infty}\left|c_{n}\right|=+\infty$ with $\sup _{n}\left|c_{n}\right|<\infty$. Then

$$
\lim _{N \rightarrow \infty}\left(\operatorname{Re} \sum_{n=1}^{N} c_{n} \exp \left(\lambda_{n} x\right)\right)=+\infty
$$

for all $x$ in some set of positive logarithmic capacity.

In this present paper we address the question of whether $\sup _{n}\left|c_{n}\right|<\infty$ is a necessary hypothesis.

In part the answer is “yes". The series $\sum_{n=1}^{\infty} 4^{n} \cos \left(2^{n} x\right)$ diverges to $+\infty$ on exactly the countable set of $x$ such that for some positive integer $n, 2^{n} x=0 \bmod 2 \pi$. However, for cosine series the theorem remains true with $q>4$ and for general series with $q>8$. The proof depends upon a new sufficient condition for a set to have positive generalized capacity.

Let $\varphi: R^{+} \rightarrow R$ be a continuous decreasing function with $\lim _{t \rightarrow 0^{+}} \varphi(t)=+\infty$. Let $\varphi$ be integrable on [0,1], and set $\Phi(t)=\int_{0}^{t} \varphi(s) d s$. We define the $\varphi$-capacity of a Borel set $E \subseteq R$ as in [1 and 2]. Let $\mu$ be a probability measure on $E$ and consider

$$
u(x)=\int_{R} \varphi(|x-y|) d \mu(y) \text { for } y \in R .
$$

$E$ is said to have positive $\varphi$-capacity if $\sup _{x \in R} u(x)<\infty$ for at least one measure $\mu$.

The theorem below concerns Cantor sets $C$ constructed as follows. $C=$ $\cap_{n=1}^{\infty}\left(\cup I_{n}\right)$ where each $I_{n}$ is a set of closed, disjoint intervals of $[0,1]$ of equal length $d_{n}$. Each interval $I$ in $I_{n}$ contains exactly $K_{n+1} \geqslant 2$ intervals from $I_{n+1}$. For ease of notation, assume also that the number of intervals in $I_{1}$ is $K_{1} \geqslant 2$. By the Lebesgue measure on $C$ we mean that probability measure $\mu$ satisfying $\mu(I)=A_{n}^{-1}$

Received by the editors January 31, 1983.

1980 Mathematics Subject Classification. Primary 42A55, 28 A12.

Key words and phrases. Lacunary series, generalized capacity, logarithmic capacity. 
for $I$ in $I_{n}$, where $A_{n}=\prod_{p=1}^{n} K_{p}$. Given any interval $J$ of length $d_{n}$, clearly no more than two intervals from $I_{n}$ can intersect it. Thus $\mu(J) \leqslant 2 A_{n}^{-1}$, an estimate which will be used later. This construction of the Cantor set $C$ is more general than that of [1] in two respects. In [1] the intervals of $I_{n+1}$ inside a single interval of $I_{n}$ are equally spaced. An examination of the proof of [1] reveals that this is unnecessary. More importantly, in [1] $K_{n+1}$ is a constant, independent of $n$.

THEOREM 2. If $\sum_{n=1}^{\infty}\left[\Phi\left(d_{n}\right) /\left(d_{n+1} \cdot A_{n+1}\right)\right]<\infty$, then $C$ has positive $\varphi$-capacity. In fact, $\sup _{x \in R} u(x)<\infty$ for $u(x)=\int \varphi(|x-y|) d \mu(y)$ with the Lebesgue measure $\mu$ on C.

Proof. Let $\mu$ be the Lebesgue measure on $C$ and let $x \in R$. Set

$$
J_{p}=\left[x-d_{p}, x+d_{p}\right] \backslash\left[x-d_{p+1}, x+d_{p+1}\right] \text { for } p \geqslant 1 .
$$

Make $J_{0}=R \backslash\left[x-d_{1}, x+d_{1}\right]$. Then

$$
\begin{aligned}
u(x) & =\int_{R} \varphi(|x-y|) d \mu(y)=\sum_{p=0}^{\infty} \int_{J_{p}} \varphi(|x-y|) d \mu(y) \\
& \leqslant \varphi\left(d_{1}\right) \mu\left(J_{0}\right)+\sum_{p=1}^{\infty} \int_{J_{p}} \varphi(|x-y|) d \mu(y) .
\end{aligned}
$$

For each $p \geqslant 1$ we estimate $\int_{J_{p}} \varphi(|x-y|) d \mu(y)$. Partition $\left[x+d_{p+1}, x+d_{p}\right]$ with $x_{0}=x+d_{p+1}, x_{1}=x+2 d_{p+1}^{p}, \ldots, x_{n_{p}}=x+n_{p} d_{p+1}$ where $x+n_{p} d_{p+1}<x+$ $d_{p} \leqslant x+\left(n_{p}+1\right) d_{p+1}$. Because $\varphi$ is decreasing and nonnegative near zero, for $p$ large

$$
\begin{aligned}
\int_{x+d_{p+1}}^{x+d_{p}} \varphi(|x-y|) d \mu(y) & \leqslant \int_{x+d_{p+1}}^{x+\left(n_{p}+1\right) d_{p+1}} \varphi(|x-y|) d \mu(y) \\
& \leqslant \sum_{j=1}^{n_{p}} \varphi\left(j d_{p+1}\right) 2 A_{p+1}^{-1}=\left(\frac{2}{d_{p+1} A_{p+1}}\right) \sum_{j=1}^{n_{p}} \varphi\left(j d_{p+1}\right) d_{p+1} .
\end{aligned}
$$

Note that $\sum_{j=1}^{n_{p}} \varphi\left(j d_{p+1}\right) d_{p+1} \leqslant \int_{0}^{n_{p} d_{p+1}} \varphi(|y|) d y$, because $\varphi$ is decreasing. This last is $\Phi\left(n_{p} d_{p+1}\right)$. Note that $n_{p} d_{p+1} \leqslant d_{p}$. Also, if $p$ is large enough, $d_{p}$ is small and $\varphi$ is positive on $\left[0, d_{p}\right]$. Then $\Phi\left(n_{p} d_{p+1}\right) \leqslant \Phi\left(d_{p}\right)$ and we have

$$
\int_{x+d_{p+1}}^{x+d_{p}} \varphi(|x-y|) d \mu(y) \leqslant \frac{2 \Phi\left(d_{p}\right)}{d_{p+1} A_{p+1}} .
$$

By treating $\int_{x-d_{p}}^{x-d_{p+1}} \varphi(|x-y|) d \mu(y)$ in a similar manner, we obtain

$$
\int_{J_{p}} \varphi(|x-y|) d \mu(y) \leqslant \frac{4 \Phi\left(d_{p}\right)}{d_{p+1} A_{p+1}} .
$$

This completes our estimate. Finally

$$
u(x) \leqslant \varphi\left(d_{1}\right) \mu\left(J_{0}\right)+\sum_{p=1}^{\infty} 4 \Phi\left(d_{p}\right) /\left(d_{p+1} A_{p+1}\right)<\infty,
$$

with a bound independent of $x$. 
Corollary 3. Let $\varphi(t)=-\log t$. Let $C$ be constructed as above; let $A_{n}, d_{n}$, and $K_{n}$ be defined as above. If for some $\alpha>0, d_{n+1} A_{n+1} \geqslant \alpha d_{n} A_{n}$, the logarithmic capacity of $C$ is positive.

Proof of Corollary. We apply the theorem. Here $\varphi(t)=-t \log t+t$. The test series is

$$
\sum_{n=1}^{\infty}\left(-d_{n} \log d_{n}+d_{n}\right) /\left(d_{n+1} A_{n+1}\right) .
$$

Because $d_{n+1} A_{n+1} \geqslant \alpha d_{n} A_{n}$, this series is dominated by

$$
\alpha^{-1} \sum_{n=1}^{\infty}\left(1-\log d_{n}\right) /\left(A_{n}\right) \text {. }
$$

By induction we have $d_{n} \geqslant \alpha^{n-1}\left(d_{1} A_{1}\right) /\left(A_{n}\right)$, so that for some constant $C$ we have

$$
1-\log d_{n} \leqslant(n-1)(-\log \alpha)-C+\log A_{n} .
$$

Note that $A_{n} \geqslant 2^{n}$. Because $(\log x) / x$ is decreasing for $x \geqslant e$, for $n \geqslant 2$ we have $\log \left(A_{n}\right) / A_{n} \leqslant \log \left(2^{n}\right) / 2^{n}$. Thus the series (2) is dominated by the convergent series (4):

$$
\alpha^{-1}\left\{\left(1-\log d_{1}\right) / A_{1}+\sum_{n=2}^{\infty}\left[((n-1)(-\log \alpha)-C+n \log 2) / 2^{n}\right]\right\}
$$

That completes the proof.

We next apply Corollary 3 to lacunary divergent trigonometric series in the proof of Theorem 4.

THEOREM 4. Let $\rho$ satisfy $0<\rho<1$. Let $\left\{\lambda_{k}\right\}_{k=1}^{\infty}$ be a lacunary sequence of positive integers such that $\lambda_{k=1} / \lambda_{k} \geqslant 8 \rho^{-1}$ for all $k$. Let $\sum_{k=1}^{\infty}\left|c_{k}\right|=+\infty$. Then there is some set $C \subset[0,2 \pi]$ of positive logarithmic capacity on which

$$
\operatorname{Re}\left\{\sum_{k=1}^{N} c_{k} \exp \left(\lambda_{k} x\right)\right\} \geqslant K_{\rho}\left(\sum_{k=1}^{N}\left|c_{k}\right|\right) \text { for all } N
$$

where $K_{\rho}=\cos (\rho(\pi / 2))$.

Proof. $C$ shall be a Cantor set as constructed above, $C=\bigcap_{n}\left(\cup I_{n}\right)$, to which we shall apply Corollary 3 . We shall have

$$
\operatorname{Re}\left\{c_{n} \exp \left(\lambda_{n} x\right)\right\} \geqslant K_{\rho}\left(\left|c_{n}\right|\right) \text { for } x \text { in } I_{n} .
$$

Each interval in $I_{n}$ shall have length $\rho(\pi / 2) \lambda_{n}$. The sets $I_{n}$ shall be chosen inductively.

Write $c_{1}=\exp \left(\varphi_{1}\right)\left|c_{1}\right|, \varphi_{1}$ real. Note that $\operatorname{Re}\left\{c_{1} \exp \left(\lambda_{1} x\right)\right\} \geqslant K_{\rho}\left|c_{1}\right|$ if and only if $\left|x-\left(\left(k \cdot 2 \pi-\varphi_{1}\right) / \lambda_{1}\right)\right| \leqslant \rho(\pi / 2) \lambda_{1}$ for some integer $k$. Let $I_{1}$ consist of one interval of length $d_{1}=\rho(\pi / 2) / \lambda_{1}$ inside $[0,2 \pi]$ such that $\operatorname{Re}\left\{c_{1} \exp \left(\lambda_{1} x\right)\right\} \geqslant K_{\rho}\left|c_{1}\right|$ on that interval.

For the inductive hypothesis assume that we have chosen $\left\{I_{k}\right\}_{k=1}^{n}$, a sequence of nested sets of intervals as described above, with the intervals of $I_{k}$ having equal 
length $d_{k}=\rho(\pi / 2) \lambda_{k}$ and $d_{k+1} \cdot A_{k+1} \geqslant(\rho / 8) d_{k} \cdot A_{k}$. We then choose $I_{n+1}$ as follows. Write $c_{n+1}$ as $\exp \left(\varphi_{n+1}\right)\left|c_{n+1}\right|$. Note that $\operatorname{Re}\left\{c_{n+1} \exp \left(\lambda_{n+1} x\right)\right\} \geqslant K_{\rho}\left|c_{n+1}\right|$ on intervals of the form

$\left[\left(p \cdot 2 \pi-\varphi_{n+1}\right) / \lambda_{n+1}-\rho(\pi / 2) / \lambda_{n+1},\left(p \cdot 2 \pi-\varphi_{n+1}\right) / \lambda_{n+1}+\rho(\pi / 2) / \lambda_{n+1}\right]$,

for arbitrary integers $p$. Let $K_{n+1}$ be the greatest integer in

$$
\rho(\pi / 2) / \lambda_{n} /\left(2 \pi / \lambda_{n+1}\right)=(\rho / 4)\left(\lambda_{n+1} / \lambda_{n}\right) \geqslant 2 \text {. }
$$

Thus each interval of $I_{n}$ (because its length is $\rho(\pi / 2) \lambda_{n}$ ) contains at least $K_{n+1}-1$ consecutive intervals of the form

$$
\left[\left(p \cdot 2 \pi-\varphi_{n+1}\right) / \lambda_{n+1},\left((p+1) 2 \pi-\varphi_{n+1}\right) / \lambda_{n+1}\right]
$$

where $p$ is an integer. Inside each interval of $I_{n}$ we take (for $I_{n+1}$ ) the initial segments of length $\rho(\pi / 2) / \lambda_{n+1}$ of those intervals of form (7). We also include in $I_{n+1}$ the last segment of length $\rho(\pi / 2) / \lambda_{n+1}$ of the last interval of form (7) inside of each interval of $I_{n}$. That completes our choice of $I_{n+1}$. There remains to be checked whether $d_{n+1} \cdot A_{n+1} \geqslant(\rho / 8) d_{n} \cdot A_{n}$. We have

$$
d_{n+1} \cdot A_{n+1}=\left(\rho(\pi / 2) / \lambda_{n+1}\right) \cdot K_{n+1} \cdot A_{n} .
$$

Recall that $K_{n+1}$ is the greatest integer in $\rho(\pi / 2) / \lambda_{n} /\left(2 \pi / \lambda_{n+1}\right)=(\rho / 4)\left(\lambda_{n+1} / \lambda_{n}\right)$. It is easy to check that the greatest integer in $(\rho / 4) y$ is no less than $(\rho / 8) y$ for $y \geqslant 8 / \rho$. It follows easily that $d_{n+1} \cdot A_{n+1} \geqslant(\rho / 8) d_{n} \cdot A_{n}$. That completes the proof of this theorem.

REMARK 5. In the case of cosine series the previous argument can be simplified because one may assume $\varphi_{k}$ is 0 for all $k$. In that case it suffices to assume that $\lambda_{k+1} / \lambda_{k} \geqslant 4 / \rho$.

\section{REFERENCES}

1. A. F. Beardon, The generalized capacity of Cantor sets, Quart. J. Math. Oxford Ser. (2) 19 (1968), 301-304.

2. S. J. Taylor, On the connexion between Hausdorff measures and generalized capacity, Math. Proc. Cambridge Philos. Soc. 57 (1961), 524-531.

3. G. Csordas, A. J. Lohwater and L. T. Ramsey, Lacunary series and the boundary behavior of Bloch functions, Michigan Math. J. 29 (1982), 281-288.

Department of Mathematics, University of Hawail at Manoa, Honolulu, Hawail 96822 